\title{
El rol de la ciudad infiltrada en la reconfiguración de la periferia metropolitana de Santiago de Chile
}

\section{The role of the infiltrated city in the configuration of Santiago's suburbs}

\author{
Gloria Naranjo Ramírez*
}

\section{INTRODUCCIÓN}

El crecimiento de las ciudades y su expansión física, unido a crecientes procesos de urbanización son una preocupación desde hace varias décadas en los países desarrollados. Estos procesos, en los países en vías de desarrollo, se han manifestado con una gran intensidad a pesar de ser más recientes, muchas veces de forma acelerada y sin control. Esta tendencia a la metropolización, según Salazar (2007), como manifestación de un proceso de concentración de la población, de actividades en áreas metropolitanas extendidas, junto con la globalización e internacionalización de los mercados y el mejoramiento de los sistemas de transporte e información, han significado profundas transformaciones en los territorios en diferentes escalas geográficas. En Chile, considerado un país en vías de desarrollo en el que se ha manifestado un modelo económico de capitalismo emergente, encontrándose en una situación intermedia de desarrollo, los impactos de las recomposiciones económicas y territoriales se convierten en un objeto de estudio para la observación de los procesos de reestructuración, especialmente en la Región Metropolitana de Santiago.

\footnotetext{
* Instituto de Geografía, Pontificia Universidad Católica de Chile, Av. Vicuña Mackenna 4860, Macul Santiago, Chile. gdnaranj@uc.cl.
} 
En este contexto, cobran un particular interés los espacios periurbanos por sus características, que los hacen territorios competitivos y estratégicos para el futuro de las metrópolis, ya que en ellos se encuentran múltiples recursos y actividades rurales complementarias al funcionamiento de estas grandes ciudades.

De acuerdo a Salazar (2007), los procesos de concentración económica y demográfica, así como los de desconcentración y de relocalización espacial acontecidos en las regiones metropolitanas en las últimas décadas, dan cuenta de un nuevo sistema de relaciones entre las sociedades y sus espacios rurales, "dando origen a una nueva ruralidad y urbanidad en las actuales sociedades post-urbanas, cada vez más móviles y globalizadas y caracterizadas por la intensidad del cambio social que se desarrolla en ellas" (Salazar, 2007:207).

Para Santiago de Chile, las transformaciones territoriales en sus espacios periurbanos y los conflictos urbano-rurales de la extensión metropolitana, se encuentran expuestas a estos cambios, pero también a las concepciones de la planificación urbana, relacionadas con las formas extendidas y fragmentadas de concebir la ciudad. Estas nuevas expresiones residenciales, con una tendencia difusa y fragmentada son parte de los resultados para esta ciudad caracterizada desde sus orígenes por la segregación socioespacial de la población, tanto dentro de la aglomeración central, como en sus espacios rurales periféricos.

Este trabajo da a conocer el impacto espacial que ha producido el DL 3.516/80 sobre subdivisión de predios rústicos en los espacios rurales contiguos a la ciudad de Santiago de Chile, espacio técnicamente denominado como periurbano. La aplicación de dicha ley en este espacio ha generado la urbanización del campo al margen de los instrumentos de ordenamiento territorial que en Chile regulan sólo los espacios urbanos. La mencionada urbanización se ha materializado, entre otras, bajo la forma de las denominadas parcelas de agrado, término creado para identificar a los lotes de hasta 0,5 hectárea que forman parte de proyectos inmobiliarios que los habitantes urbanos adquieren como segunda residencia o como residencia definitiva en busca de una mejor calidad de vida. Dado su tamaño, no es posible realizar en ellas una actividad agrícola rentable y quienes las adquieren no tienen vocación agrícola, por lo que terminan formando parte de la ciudad infiltrada.

A través de diferentes estudios de caso realizados en comunas de la periferia de la ciudad de Santiago, especialmente en la provincia de Chacabuco, en la Región Metropolitana de Santiago, se intenta dar respuesta a interrogantes tales como: ¿Qué superficie ha sido afectada por el DL 3.516/80 en 
las diferentes comunas que se han estudiado?, ¿Cuánto ha avanzado la ciudad infiltrada sobre los suelos de aptitud agrícola de la periferia de la ciudad de Santiago de Chile? ¿Cuál de las comunas estudiadas es la más afectada? ¿Cuál es el papel que cumplen los instrumentos de ordenamiento territorial, de sesgo urbano, en los espacios rurales contiguos a la ciudad?, ¿Cómo ha cambiado el paisaje de la periferia de Santiago de Chile por efecto del DL 3.516/80?

Como hipótesis, se postula que el proceso de puesta en marcha del Plan Regulador Metropolitano de Santiago generó efectos en el territorio externo al área que regula el instrumento de ordenamiento territorial. Se utiliza el artificio legal que representa el DL 3.516/80 para la subdivisión de los territorios exteriores al alcance del Plan Regulador Metropolitano de Santiago, a la espera de la agilización del mercado de la tierra. El proceso de especulación en el mercado de la tierra se desata con la aparición del PRMS y se prueba su existencia a través del análisis del estado de consolidación de los predios acogidos al DL 3.516.

Desde el punto de vista metodológico, el trabajo consideró una serie de etapas, tales como la recopilación bibliográfica de información, principalmente aportada por el Servicio Agrícola Ganadero y Oficinas del Conservador de Bienes Raíces, la cual permitió definir el período de estudio para cada comuna, el número de predios, su localización y la superficie afectada por el DL 3.516/80. Esta información fue complementada con entrevistas a actores locales claves en temas afines a esta investigación, salidas a terreno, elaboración y análisis de cartografía, fotomosaicos y fotografías aéreas, para el levantamiento de información del contexto físico del área de estudio, la determinación del sistema predial y capacidad de uso del suelo respectivamente. El tratamiento de la información en formato digital se realizó mediante el uso del SIG ArcView 3.3.

Cabe destacar que en el proceso de análisis de la información, se consideran como "subdivisiones consolidadas" todos aquellos predios individualizados por sus roles de propiedad, que a la fecha están con sus subdivisiones certificadas por el Servicio Agrícola Ganadero y que presentan sus obras de urbanización iniciadas, entendiéndose por tales la construcción de caminos, casa, red de agua potable, alcantarillado, alumbrado público, entre otros. Se consideran como "subdivisiones no consolidadas" a todos aquellos predios individualizados por sus roles de propiedad que a la fecha están con sus subdivisiones certificadas por el Servicio Agrícola Ganadero y que no presentan obras de urbanización 


\section{LA EXPANSIÓN DEL GRAN SANTIAGO}

La ciudad de Santiago presenta durante el último siglo un fuerte crecimiento en expansión. Este fenómeno es de gran relevancia por sus efectos políticos, económicos y sociales, más aun cuando esta expansión se concentra principalmente en el centro del país.

La ciudad de Santiago, por su gran dimensión territorial y demográfica, se considera de carácter único y metropolitano; concentra una población de 5.822.316 habitantes a 2002 (INE, 2002), se ubica en la cuenca del río Maipo, cuya superficie aproximada es de $14.600 \mathrm{~km}^{2}$, ocupando la superficie construida de la ciudad de Santiago 58.300 hectáreas (Arenas e Hidalgo, 2002). Pero el crecimiento de la ciudad de Santiago se ha intensificado a partir de la segunda mitad del siglo pasado. Es así como en el año 1960, la ciudad se ha expandido notoriamente en relación a la década anterior. Su superficie se ha incrementado en $58,14 \mathrm{~km}^{2}$, con un crecimiento de 3,7 por ciento, alcanzando a 21.810,03 hectáreas (Miranda, 1997). Cabe destacar que en este período se habían incorporado al Gran Santiago 7.622,9 hectáreas de suelos arables de capacidad de uso I, II, III y IV (Brignardello y Georgudis, 1997). Estos últimos corresponden a terrenos adecuados para cultivos, desde aquellos sin limitaciones, hasta los que presentan limitaciones severas.

Por otra parte, según Brignardello y Georgudis (1997), en 1960 la ciudad contaba con 1.907.378 habitantes, con un crecimiento anual del 3,7 por ciento, lo que definía una densidad poblacional de $87,6 \mathrm{hab} / \mathrm{ha}$. Con respecto al total nacional, que entonces ascendía a la cifra de 7.374.115 habitantes, a este año, representaba el 25,9 por ciento de la población nacional (Miranda, 1997).

Al año 1970, la expansión urbana de Santiago se expresaba con fuerza hacia todos los sectores del valle. Es así como a este año la superficie urbana alcanzaba a 29.596,64 hectáreas, presentando un crecimiento de su superficie del orden del 2,0 por ciento. La población a este año alcanzaba a los 2.730.000 habitantes, con un crecimiento anual del 3,5 por ciento, siendo su densidad de 92,2 hab/ha (Brignardello y Georgudis, 1997). Para ese mismo año, la población del país alcanzaba a los 8.884.768 habitantes, por lo que la población del Gran Santiago representaba en ese entonces al 30,7 por ciento. A esa fecha el país presenta una alta urbanización, alcanzando el 75,1 por ciento de población urbana. Se le han anexado las ciudades de Puente Alto y San Bernardo por procesos espaciales de conurbación.

En 1970 se habían incorporado a la ciudad 9.244,2 hectáreas de suelos arables de capacidad de uso I, II, III y IV. En esta década se da inicio al actual proceso de regionalización del país, lo cual permite un mejor manejo 
administrativo del territorio. Las comunas del Gran Santiago se modifican en tamaño y se crean otras, facilitando la implantación de políticas urbanas en la ciudad.

Entre las políticas de desarrollo urbano aplicadas en el país, destaca la Ley General de Urbanismo y Construcciones del año 1975, que establece cuatro niveles de planificación: nacional, regional, intercomunal y comunal. Además, establece la aplicación del límite urbano y el uso del suelo de los centros poblados en un plan regulador.

El acelerado crecimiento demográfico y territorial de Santiago determinó una gran preocupación de las autoridades, por lo que en 1975 se dictó un Decreto de congelación de los límites urbanos para todas las ciudades del país, que pretendía detener el crecimiento y en algunos casos, revertir el proceso, recobrando tierras con destino agrícola. Con este hecho se produce una gran alza de los valores del suelo urbano. En 1979 el Gobierno pone fin a esta política e inicia una apertura del proceso de desarrollo urbano de acuerdo al nuevo modelo económico social de mercado implantado, lo que significó un vuelco de los lineamientos anteriores. Como consecuencia de esta disposición, la expansión urbana de Santiago se acelera, provocando un gran crecimiento, especialmente de las comunas del sector oriente. Se incorporan al área urbana alrededor 8.000 hectáreas a través de loteos, subdivisiones y parcelaciones localizados en el área de expansión urbana.

En el año 1980, la superficie urbana del Gran Santiago alcanzaba a $38.649,15$ hectáreas, con un crecimiento del 2,7 por ciento. Se han incorporado $5.867,7$ hectáreas de suelos arables a la ciudad. La población, por otra parte, alcanzaba una cifra de 3.650.000 habitantes, con un crecimiento del 3,0 por ciento anual y una densidad de $94,4 \mathrm{hab} / \mathrm{ha}$. Cabe destacar que el descenso de la densidad poblacional se debe en gran parte al efecto producido por la nueva política de desarrollo urbano que genera una acelerada expansión de la ciudad, ya que postulaba que el suelo urbano no es un recurso escaso, por lo que el uso del suelo queda definido por su mayor rentabilidad.

La población nacional al año 1982 era de 11.329 .736 habitantes, por lo que la población de Santiago con 3.902.356 habitantes, representa el 34,4 por ciento del total nacional.

Según los datos aportados por el Censo de 1992, la población del Gran Santiago era de 4.754 .901 habitantes. El total nacional para ese mismo año fue de 13.348.401 habitantes, por lo tanto, Santiago concentra en ese entonces el 35,6 por ciento de la población nacional.

Al año 2002, la ciudad de Santiago tiene una población que alcanza a 5.822.316 habitantes, cifra que representa el 38,5 por ciento de la población 
nacional y ostenta una superficie construida que bordea las 60.000 hectáreas.

La creciente expansión urbana experimentada por la ciudad de Santiago se refleja en las cifras de concentración de la población respecto al total de población nacional. En el año 1960, Santiago concentraba el 25,9 por ciento de la población nacional, en 1970 el 31,8 por ciento, en 1982 el 34,4 por ciento, en 1992 el 35,6 por ciento y en 2002 el 38,5 por ciento. Como puede apreciarse, son cifras crecientes a pesar de los innumerables esfuerzos emprendidos por los gobiernos de turno tendientes a la descentralización.

\section{EL CONTEXTO DE LA EXPANSIÓN}

La ciudad de Santiago, al igual que otras ciudades latinoamericanas, presenta una nueva configuración territorial, producto de una acelerada expansión acontecida durante las últimas décadas. Esta configuración sería propia de las áreas metropolitanas de economías emergentes, que, son los lugares que ofrecen una mayor acumulación inicial de capital físico, capital humano, conocimientos y técnicas; que muestran también una mayor atracción para los movimientos nacionales e internacionales de capital; elementos que estarían incidiendo en los factores de localización que favorecen el comando del nuevo poder económico, político y social, así como los servicios más avanzados y lo más dinámico e innovador de las comunicaciones. Estas áreas metropolitanas se consolidan como sede principal de un conjunto de funciones y actividades que corresponden a los atributos de una ciudad global (Sassen, 1991), tales como las funciones de dirección, gestión, coordinación y control de las principales estructuras empresariales que comandan las dinámicas de acumulación, así como la articulación de la economía global; las actividades básicas del terciario avanzado; el núcleo más dinámico e innovador de la nueva industria y el mercado para la parte más relevante de los productos globales (De Mattos, 1998).Por otra parte, la localización en estas metrópolis, determina que sea en ellas donde se encuentren también los mercados de trabajo más importantes.

La manifestación territorial de estos procesos de crecimiento metropolitano se expresan en una nueva configuración de la ciudad, propia de la época de la globalización y de la desregulación, para constituir las metrópolis-regiones en constante proceso de expansión territorial, con una incontrolable tendencia hacia la suburbanización y la periurbanización a partir de los núcleos urbanos originales, ocupando áreas rurales próximas y sobrepa- 
sando los límites urbanos definidos con anterioridad ${ }^{1}$. A este tipo de configuración urbana se le han dado distintas denominaciones, tales como "área metropolitana", "región metropolitana", "ciudad región", "región urbana", "megalópolis", "metápolis" (Ascher, 1995), "ciudad global" (Sassen, 1991). Cada término ha sido usado para referir alguna característica en particular, sin embargo, todos ellos aluden desde el punto de vista de la forma urbana, a una "metrópoli-región policéntrica, que en su persistente expansión se va configurando como un verdadero archipiélago urbano de fronteras difusas" (Ferrao, 1992; Dollfus, 1994).

Pero esta forma de expansión urbana, en el período desarrollista ya había comenzado a perfilarse. Según De Mattos (2005), al culminar dicha fase (fue especialmente en las décadas de los años cincuenta y sesenta en que la expansión urbana alcanzó mayor impulso), ya se vislumbraba la tendencia hacia la formación de una ciudad-región, cuya dinámica expansiva terminaría por anexar a algunos centros urbanos aledaños con funcionamiento relativamente independiente, tales como San Bernardo, Puente Alto, al mismo tiempo que articularía al funcionamiento metropolitano a otros bajo la modalidad de ciudades satélites o ciudades dormitorio, tales como Melipilla, Talagante, Colina. Es entonces la intensificación de la urbanización capitalista lo que sí es nuevo, como producto de un continuo proceso de concentración de procesos productivos, población, necesidad de proximidad entre ellos y al mismo tiempo, y con el lugar donde se encuentran las mayores economías de aglomeración.

La ciudad de Santiago, en su expansión, ha incorporado centros urbanos aledaños y ocupado de manera parcial e incompleta las áreas rurales adyacentes bajo la forma de un espacio híbrido, compleja mezcla de lo urbano y lo rural. En palabras de De Mattos (1995:39), "en esta aglomeración, sin duda mucho más extendida, más imprecisa y más difícil de delimitar y de controlar, ya no es tan claro lo que es ciudad y lo que no lo es, aun cuando en su ámbito funcional los modos de vida urbanos se impongan por doquier". En ellos, la planificación urbana no juega en la práctica rol alguno.

\footnotetext{
${ }^{1}$ Investigaciones sobre «grandes ciudades regiones», entre las que se incluyeron dos latinoamericanas (Santiago de Chile y Sao Paulo en Brasil) confirman que «en todas estas ciudades, el modelo predominante de crecimiento físico ha sido la dispersión desde el centro histórico y los anillos interiores adyacentes hacia distantes espacios abiertos y tierra agrícola». (Lincoln Institute, 1996:3).
} 
EL MARCO LEGAL

Hay dos hechos concretos que permitieron el desarrollo de esta nueva forma de suburbanización. Estos son el Decreto Supremo 420 del Ministerio de Vivienda y Urbanismo (MINVU) de 1979, que eliminó el límite urbano y el Decreto Ley 3.516/80 sobre subdivisión de predios rústicos, que dio lugar a la ciudad informal, extraoficial o infiltrada en torno a las áreas urbanas.

El Decreto Supremo 420 del MINVU se relaciona con la Política de Desarrollo Urbano aprobada en 1979, en la que se asume el suelo como un bien que se transa en forma abierta en el mercado, sin consideración alguna respecto de su aptitud y limitaciones naturales. Tanto el suelo urbano como el rural con potencialidad urbana, dejan de ser recursos sujetos a planificación rígida, regulándose por las condiciones de dicho mercado. En consecuencia, constituye un objetivo de esa Política, eliminar las restricciones que impidan el crecimiento natural de las áreas urbanas. Así, las ciudades pueden crecer sobre los suelos agrícolas mientras las condiciones imperantes en el mercado creen incentivos y provean los recursos necesarios para su urbanización.

El Decreto Ley 3.516/802 sobre subdivisión de predios rústicos permite la subdivisión de cualquier terreno localizado en áreas rurales, en hasta 0,5 hectárea, previa aprobación del Servicio Agrícola Ganadero (SAG) dependiente del Ministerio de Agricultura. Las subdivisiones en áreas rurales con fines urbanos (plantas industriales, campamentos mineros, núcleos turísticos, entre otros), se rigen por el Art. 55 de Ley General de Urbanismo y Construcciones ${ }^{3}$ (LGUC), que establece la obligación de cumplir con exigencias de urbanización que son financiadas por el dueño.

Entre los atributos del DL 3.516/80, cabe destacar que las subdivisiones a que da lugar su aplicación no están sometidas a ningún tipo de exigencias de urbanización, como tampoco a ningún trámite de aprobación, salvo los requerimientos estipulados por la resolución No 1.885 de agosto de 19954. Prohíbe destinar las parcelaciones resultantes a fines residenciales (urbanos) y los predios a que de lugar una subdivisión, quedarán sujetos a la prohibición de cam-

\footnotetext{
2 Publicado en el Diario Oficial No 30.829 de 1 de diciembre de 1980.

${ }^{3}$ La Ley General de Urbanismo y Construcciones regula aspectos relacionados con la construcción, urbanización y planificación urbana. Pretende orientar y regular el desarrollo de los centros urbanos en función de una política nacional, regional y comunal de desarrollo socioeconómico. Esta se realiza en cuatro niveles de acción, que son el nacional, regional, intercomunal y comunal.

${ }^{4}$ Resolución $N^{\circ} 1.855$ del 03/08/1995, establece antecedentes requeridos para la subdivisión de predios rústicos.
} 
biar su destino, salvo en los casos en que se solicite el cambio de uso de suelo a la Comisión Interminsterial Ministerio de Vivienda y Urbanismo-Ministerio de Agricultura. De esta forma, cuando no se consulta cambio de uso de suelo a otro no residencial, la ley permite la construcción de dos casas en cada predio, lo que de hecho posibilita una suburbanización de baja densidad. Así, se cambia el destino y función de estos espacios, se produce un claro deterioro paisajístico, además del impacto social, económico y cultural en la población local.

Sin embargo, el DL 3.516/80 contiene una contradicción interna. Al no discriminar sobre la calidad de los suelos, disponibilidad de riego, topografía, clima, ubicación, lo reducido de la superficie impide una explotación agrícola rentable, lo que hace que la superficie de los predios sea incompatible con el destino pretendido. Cabe recordar que uno de los objetivos de esta ley pretendió beneficiar a los asignatarios de reforma agraria luego de desatado el proceso de venta de tierras asignadas. Se formula esta ley como una estrategia para impedir que este segmento de campesinos se quedara sin tierra, pudiendo conservar al menos media hectárea, en la que podrían desarrollar una agricultura de autoconsumo.

Como consecuencia, se ha utilizado el DL 3.516/80 para fines urbanos, pues ha producido un fenómeno de avance de la urbanización sobre espacios rurales carentes de regulación, bajo la figura de las "parcelas de agrado5", creación de empresas inmobiliarias que compran tierras a bajo precio y las ofrecen, logrando captar las necesidades de los habitantes de la ciudad que quieren escapar de las desventajas que representa la vida urbana (altos niveles de contaminación, congestión e inseguridad). Lo anterior, unido a los altos precios del suelo urbano, que ha generado una demanda explosiva por habitar fuera de la ciudad. Así, se "hace ciudad" fuera de los límites urbanos que son determinados por los planos reguladores. El resultado es el surgimiento de una suburbanización informal incompleta que, a modo de relleno, va cubriendo los espacios rurales de las comunas de la Región Metropolitana de Santiago desligada de los instrumentos de planificación, dando lugar a la ciudad infiltrada.

\footnotetext{
${ }^{5}$ Nota de la autora: Parcelas de agrado es el nombre con que se ha denominado a los predios de hasta 0,5 ha, que forman parte de proyectos inmobiliarios y que los habitantes urbanos adquieren como segunda residencia o como residencia definitiva en busca de una mejor calidad de vida. Por su tamaño, no es posible realizar en ellas una actividad agrícola rentable y quienes las adquieren no tienen vocación agrícola, por lo que terminan formando parte de la ciudad informal.
} 
Otro elemento de relevancia es el Plan Regulador Metropolitano de Santiago, instrumento de ordenamiento territorial que fija el límite urbano y define los usos del suelo en la ciudad de Santiago y que ve la luz pública en el año 1994. No obstante, desde este hacia la periferia, en los espacios rurales carentes de regulación específica, es posible aplicar el DL 3.516/80 y constituir las parcelas de agrado y con ellas contribuir al establecimiento de una suburbanización informal, "extraoficial", dando lugar a la ciudad infiltrada.

\section{LAS CONSECUENCIAS: GENERACIÓN DE ÁREAS RESIDENCIALES EN LOS ESPACIOS RURALES}

La expansión de la ciudad de Santiago está dando origen a una suburbanización de la periferia muy diferente a la tradicional. Hasta hace unas décadas, la periferia de Santiago se caracterizaba por ser básicamente residencial, continua al área tradicional consolidada, de densidad homogénea y funcionalmente dependiente de la ciudad central. Según Greene y Soler (1995:53), "a partir de los años ochenta, la periferia ha cambiado su vocación, su relación con la ciudad consolidada y su forma de gestión": desde una vocación orientada principalmente a la función residencial, pasa a ser multifuncional; desde una relación de dependencia con la ciudad consolidada, propia de un sistema urbano monolítico, surgen periferias con distintos niveles de autonomía y continuidad espacial; desde una gestión conducida por instrumentos formales de planificación urbana, se pasa a una casi total desregulación. Estos nuevos tipos de suburbanización dan cuenta, según los autores, del debilitamiento de la planificación urbana que se produjo a partir de los años setenta, cuando el país adoptó el modelo neoliberal de desarrollo. De esta manera, la falta de regulación de los espacios rurales en los que el DL 3.516/80 se hizo aplicable, ha originado un crecimiento extraoficial de la ciudad de Santiago, dando cuenta de lo que algunos autores han denominado "la ciudad informal", "ciudad extraoficial". Sin embargo, no resulta ser ni lo uno ni lo otro, pues se ampara en leyes que han hecho posible su existencia.

Entre las consecuencias de este hecho, destacan:

- Para la agricultura: la expansión se ha desarrollado sobre suelos de uso agrícola desprotegidos legalmente provocando un cambio en el uso del suelo que es irreversible. Se suma a esto el efecto que se produce a la actividad agrícola por una disminución de la superficie de cultivo, las repercusiones posibles en el abastecimiento de productos agrícolas a los 
mercados urbanos, mano de obra ociosa, mayor dependencia de otros mercados, entre otras.

- Para el Estado: representa un problema para los municipios afectados, ya que deberán financiar la dotación de servicios e infraestructura en estos espacios. Esto, sin duda, resiente los presupuestos municipales.

- Para los habitantes rurales tradicionales: la llegada de nuevos residentes termina por afectar sus modos de vida ligados al campo, ya que cambian su entorno y la forma en que ellos se relacionan con él. Además afecta sus sentimientos de identidad con su lugar.

- Para toda la población: la expansión representa la llegada de ciertos problemas urbanos, tales como congestión, contaminación (basura, polvo en suspensión por el paso de vehículos en caminos sin pavimentar, entre otros), inseguridad.

- Para el territorio: se genera un conflicto entre su vocación y su destino.

\section{ACCIONES PARA EL CONTROL DEL PROCESO DE EXPANSIÓN}

Lo descrito en el apartado anterior es una situación que se ha verificado en el área de expansión de la ciudad de Santiago, una faja de gran dinamismo demográfico y mutaciones en el mercado de tierras.

El DL 3.516/80 entra en vigencia en el año 1980 y se aplica en la Provincia de Chacabuco hasta el año 1997.

En 1994 se instaló el Plan Regulador Metropolitano de Santiago (PRMS), instrumento de ordenamiento territorial que fija el límite urbano y, con ello, define los territorios en los cuales se hace aplicable el DL 3.516/80. Cabe destacar que incorpora todas las comunas de la provincia de Santiago, además de San José de Maipú, Pirque y Calera de Tango.

A partir del año 1998 se incorporan los territorios de la provincia de Chacabuco (comunas de Lampa, Colina y Tiltil) al PRMS, instrumento que prohíbe explícitamente las subdivisiones prediales en virtud de esta ley dentro de la superficie provincial. Durante los 17 años que estuvo en vigencia, que coincidió además con el momento en que Santiago entró en un acelerado proceso de expansión física, logró transformar las áreas rurales de la periferia de Santiago correspondientes a esta provincia, contribuyendo al surgimiento de la ciudad infiltrada, al margen de los instrumentos de ordenamiento territorial. Esto queda en evidencia al constatar que, antes de la incorporación de la provincia de Chacabuco al PRMS, se subdividieron 122.542 hectáreas, cifra que representa casi el 60 por ciento de su superficie. 
En 2006 se incorporan al PRMS todas las comunas de las provincias de Talagante y Melipilla, además de las comunas de Buin y Paine, quedando, de esta manera, todas las comunas de la Región Metropolitana de Santiago sujetas a las normas de uso del suelo que establece. Entre sus disposiciones destaca que permitirá que toda la Región Metropolitana de Santiago pueda ser urbanizada a partir de Proyectos de Desarrollo Urbano Condicionado (PDUC); podrá recibir en los territorios que regula más de 1.300.000 habitantes más en los próximos 12 años, garantizando un crecimiento mucho mejor planificado a través de los Planes Reguladores Comunales de cada comuna y de los PDUC; define áreas de protección, 300.000 hectáreas de valor natural; entrega herramientas de desarrollo a los gobiernos locales; normará el crecimiento fuera de los límites urbanos, donde se urbaniza vía DL 3.516/80. Sin embargo, su aparición ha generado controversias, ya que se postula que mantiene ciertas contradicciones entre el Plan de Descontaminación de la ciudad de Santiago y el Ministerio de Vivienda y Urbanismo (MINVU) que pretende seguir agrandando la ciudad; da prioridad a los desarrolladores inmobiliarios en vez de a los agricultores; representa un negocio para las inmobiliarias al aumentar la oferta de suelo urbanizable, entre otras.

Área de estudio: la Provincia de Chacabuco en el CONTEXTo Metropolitano

El área de estudio corresponde a la provincia de Chacabuco, localizada al noroeste en la Región Metropolitana de Santiago. Es una de las seis provincias que componen la Región y está integrada por las comunas de Colina, Lampa y Tiltil. Cuenta con una superficie de $2.071,2 \mathrm{~km}^{2}$, cifra que la ubica en el tercer lugar luego de las provincias Cordillera y Melipilla y que corresponde al 14 por ciento de la superficie regional y al 0,20 por ciento de la superficie nacional. El 46,8 por ciento de su superficie corresponde a la comuna de Colina, con $971 \mathrm{~km}^{2}$, el 31,4 por ciento corresponde a la comuna de Tiltil con $650 \mathrm{~km}^{2}$ y el 21,6 por ciento a la comuna de Lampa, con $450 \mathrm{~km}^{2}$.

La capital de la provincia de Chacabuco es Colina.

El territorio de la provincia de Chacabuco está estructurado, desde el punto de vista de las geoformas, por la Cordillera de Los Andes, la Depresión Intermedia y la Cordillera de la Costa. Pertenece a la hoya hidrográfica del río Mapocho, afluente del Maipo, y es drenado por el sistema fluvial LampaColina. 


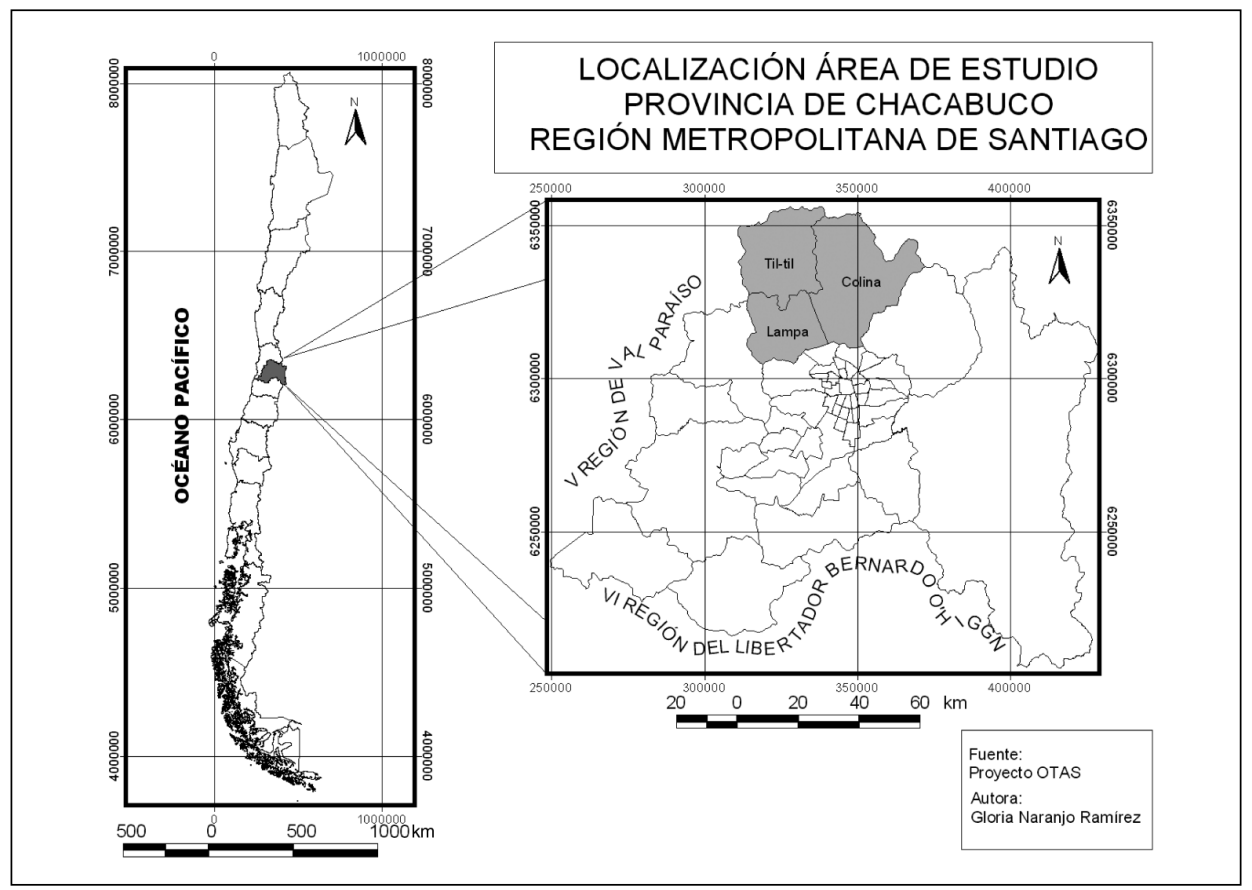

Fuente: elaborado por la autora.

MaPa 1. Localización del área de estudio

El Cordón de Chacabuco marca el límite de la cuenca por el norte y la separa del valle del río Aconcagua. El Cordón de cerros de El Manzano, en el sector sur de la provincia, la divide en dos sectores de diferentes altitudes, originándose cuencas distintas. El sector norte está integrado por la Cuenca del estero Tiltil y la Cuenca de los esteros Chacabuco y Peldehue, que constituyen un valle de potencial agrícola y frutícola. El sector sur corresponde al valle de los esteros Lampa y Colina, que tienen diversas potencialidades para la actividad agropecuaria y ciertas limitaciones naturales debido a deficiencias de drenaje en el sector de Lampa.

La mayor parte de los suelos de la provincia de Chacabuco corresponden a no arables, con un 73,62 por ciento del total de superficie. De manera que, solo un tercio de la superficie provincial corresponde a suelos arables. De las tres comunas, Tiltil es la que ostenta una mayor proporción de suelos no arables, con un 83,9 por ciento de su superficie, en tanto la comuna de Lampa 
cuenta con el mayor porcentaje de suelos arables, siendo de 37 por ciento. Ver tabla a continuación.

Como es posible apreciar en la tabla 1 , los suelos aptos para la agricultura son escasos en la provincia de Chacabuco, y aquellos con menores limitaciones para esta actividad, corresponden a alrededor del 10 por ciento de la superficie de cada comuna.

TABLA 1

CAPACIDAD DE USO DEL SUELO. PROVINCIA DE CHACABUCO

\begin{tabular}{ccccccc}
\hline & Colina & \multicolumn{3}{c}{ Lampa } & \multicolumn{3}{c}{ Tiltil } \\
\hline $\begin{array}{c}\text { Capacidad } \\
\text { de uso }\end{array}$ & $\begin{array}{c}\text { Superficie } \\
\text { (ha) }\end{array}$ & Porcentaje & $\begin{array}{c}\text { Superficie } \\
\text { (ha) }\end{array}$ & Porcentaje & $\begin{array}{c}\text { Superficie } \\
\text { (ha) }\end{array}$ & Porcentaje \\
\hline I & $6.152,87$ & 9,06 & $1.146,62$ & 2,5 & $2.427,47$ & 3,71 \\
II & $5.340,21$ & 7,86 & $2.942,97$ & 6,5 & $4.855,15$ & 7,42 \\
III & $5.077,75$ & 7,47 & $7.037,51$ & 15,6 & $2.146,87$ & 3,28 \\
IV & $2.800,32$ & 4,12 & $5.443,36$ & 12,1 & $1.090,15$ & 1,67 \\
Arables & $19.371,15$ & 28,51 & $16.570,46$ & 36,82 & $10.519,64$ & 16,8 \\
V & - & - & 837,49 & 1,9 & - & - \\
VI & $4.143,05$ & 6,10 & $2.091,96$ & 4,6 & $3.841,20$ & 5,87 \\
VII & $26.316,01$ & 38,74 & $18.052,83$ & 40,0 & $21.979,68$ & 33,61 \\
VIII & $16.688,88$ & 24,57 & $6.674,43$ & 14,8 & $29.053,49$ & 44,42 \\
No Arables & $47.147,94$ & 69,40 & $27.656,71$ & 61,46 & $54.874,37$ & 83,90 \\
Otros & $1.417,89$ & 2,09 & 840,09 & 1,86 & - & - \\
Total & $67.936,98$ & 100 & $45.067,26$ & 100 & $65.394,01$ & 100 \\
\hline
\end{tabular}

Fuente: elaborado por la autora a partir de CIREN-CORFO 1997.

En cuanto a población, la provincia de Chacabuco, según el Censo 2002, cuenta con 132.798 habitantes, de los cuales el 74,7 por ciento corresponde a población urbana y el 25,3 por ciento a población rural. De las tres comunas que componen la provincia, Colina, es la que registra un mayor monto de población, alcanzando a 77.815 habitantes, cifra que representa el 58,6 por ciento de la población provincial, correspondiendo en un 80,7 por ciento a población urbana y en un 19,3 por ciento a población rural. Luego está Lampa, con 40.228 habitantes, el 30,3 por ciento de la población provincial, de los cuales, el 70,18 por ciento corresponde a población urbana y el 29,82 
por ciento a población rural. Finalmente, Tiltil, con 14.755 habitantes, sólo representa el 11,1 por ciento de la población de la provincia de Chacabuco, correspondiendo el 55,3 por ciento a población urbana y el 44,3 por ciento a población rural. Como se puede apreciar en las cifras dadas a conocer, La comuna de Colina reúne a más de la mitad de la población de la provincia, la comuna de Lampa a un tercio, en tanto Tiltil sólo tiene un décimo de la población provincial.

\section{Efectos del DL 3.516 en la Provincia de Chacabuco}

\section{Impacto espacial del DL 3.516 en la provincia de Chacabuco}

En la provincia de Chacabuco el DL 3.516 comienza a aplicarse en el año 1980. Sin embargo, es a partir de 1994, año en que se da a conocer el Plan Regulador Metropolitano de Santiago (PRMS), instrumento de ordenamiento territorial que fija el límite urbano, cuando se comienza a hacer un uso más destacado de él. Se aplica en los territorios de la provincia de Chacabuco hasta el año 1997, pues en 1998 se hace efectiva la incorporación de esta provincia al PRMS, quedando explícitamente prohibidas las subdivisiones prediales en virtud de esta ley. Por lo tanto, se pudo hacer uso de ella durante 17 años, período en el que produjo notables cambios en el paisaje. Afectó a 1.592 predios, que abarcan una superficie de $122.542,23 \mathrm{ha}$, cifra que representa el 59,16 por ciento de la superficie provincial. La comuna más afectada fue Colina, con el 80,04 por ciento de su superficie, en tanto Lampa y Tiltil tienen superficies afectadas de alrededor del 40 por ciento.

En la comuna de Colina el DL 3.516 afecta a un total de 837 predios, cifra que involucra a $77.738,43$ ha y que, como ya se ha dicho, equivale al 80,04 por ciento de la superficie comunal. En la comuna de Lampa, el total de predios afectados es de 629, que corresponde a 18.120,12 ha, cifra que involucra al 40,26 por ciento de la superficie comunal. Finalmente en la comuna de Tiltil el DL 3.516 afecta a 126 predios, equivalentes a 26.683 ha, que representan el 41,05 por ciento de la superficie comunal. Ver tabla 2.

En los años previos a la aparición del PRMS, es decir, en el período comprendido entre los años 1980 y 1993 la superficie afectada por el DL 3.516 es muy marginal, con cifras de 3,58 por ciento para Lampa y de 1,76 por ciento para Colina, destacando el caso de la comuna de Tiltil en que no se acoge predio alguno a esta ley en este período. 
TABLA 2

PREDIOS ACOGIDOS AL DL 3.516 EN LAS COMUNAS DE LA REGIÓN METROPOLITANA

\begin{tabular}{|c|c|c|c|c|c|c|c|}
\hline & Colina & Lampa & Tiltil & $\begin{array}{c}\text { Total } \\
\text { provincia }\end{array}$ & Paine & $\begin{array}{l}\text { Calera } \\
\text { de Tango }\end{array}$ & El Monte \\
\hline $\begin{array}{l}\text { Número } \\
\text { de predios } \\
\text { acogidos }\end{array}$ & 837 & 629 & 126 & 1.592 & 783 & 375 & 244 \\
\hline $\begin{array}{l}\text { Número } \\
\text { de lotes } \\
\text { generados }\end{array}$ & 48.641 & 14.642 & 10.243 & 73.526 & 18.180 & $\mathrm{~s} / \mathrm{i}$ & 2.967 \\
\hline Superficie & $77.738,43$ & $18.120,12$ & $26.638,68$ & $122.542,20$ & $20.807,91$ & 3.258 & $5.400,8$ \\
\hline $\begin{array}{l}\text { Porcentaje } \\
\text { superficie } \\
\text { comunal }\end{array}$ & 80,04 & 40,26 & 41,05 & 59,16 & 30,7 & 44 & 45,7 \\
\hline $\begin{array}{l}\text { Superficie } \\
\text { consolidada }\end{array}$ & $49.829,09$ & $2.695,85$ & 211,34 & $52.736,28$ & $2.659,02$ & $1.395,2$ & 1.721 \\
\hline $\begin{array}{l}\text { Porcentaje } \\
\text { superficie } \\
\text { acogida }\end{array}$ & 64,09 & 14,87 & 0,79 & 43,03 & 26,22 & 42,8 & 31,8 \\
\hline
\end{tabular}

Fuente: datos elaborados por la autora sobre la base de CIREN 1997, archivos SAG y Conservador de Bienes Raíces

A continuación, se presenta como ejemplo el caso de la comuna de Colina en la provincia de Chacabuco, para tener una visión de la distribución de los predios acogidos al DL 3.516/80 en este territorio. Esta comuna destaca por la intensidad en que ha sido afectada por el proceso de subdivisión predial a través de esta ley. Al observar el Mapa 2 se puede verificar que una importante proporción de superficie comunal se vio afectada por el DL 3.516/80. Los predios afectados se localizan preferentemente en el área centro y sur de la comuna, en torno a los centros poblados de Colina, Esmeralda, Lo Pinto y Liray. Los 837 predios afectados por esta ley darían lugar a 48.641 lotes, que son nuevos predios una vez que se consolidaran todos los proyectos de parcelación aprobados. 


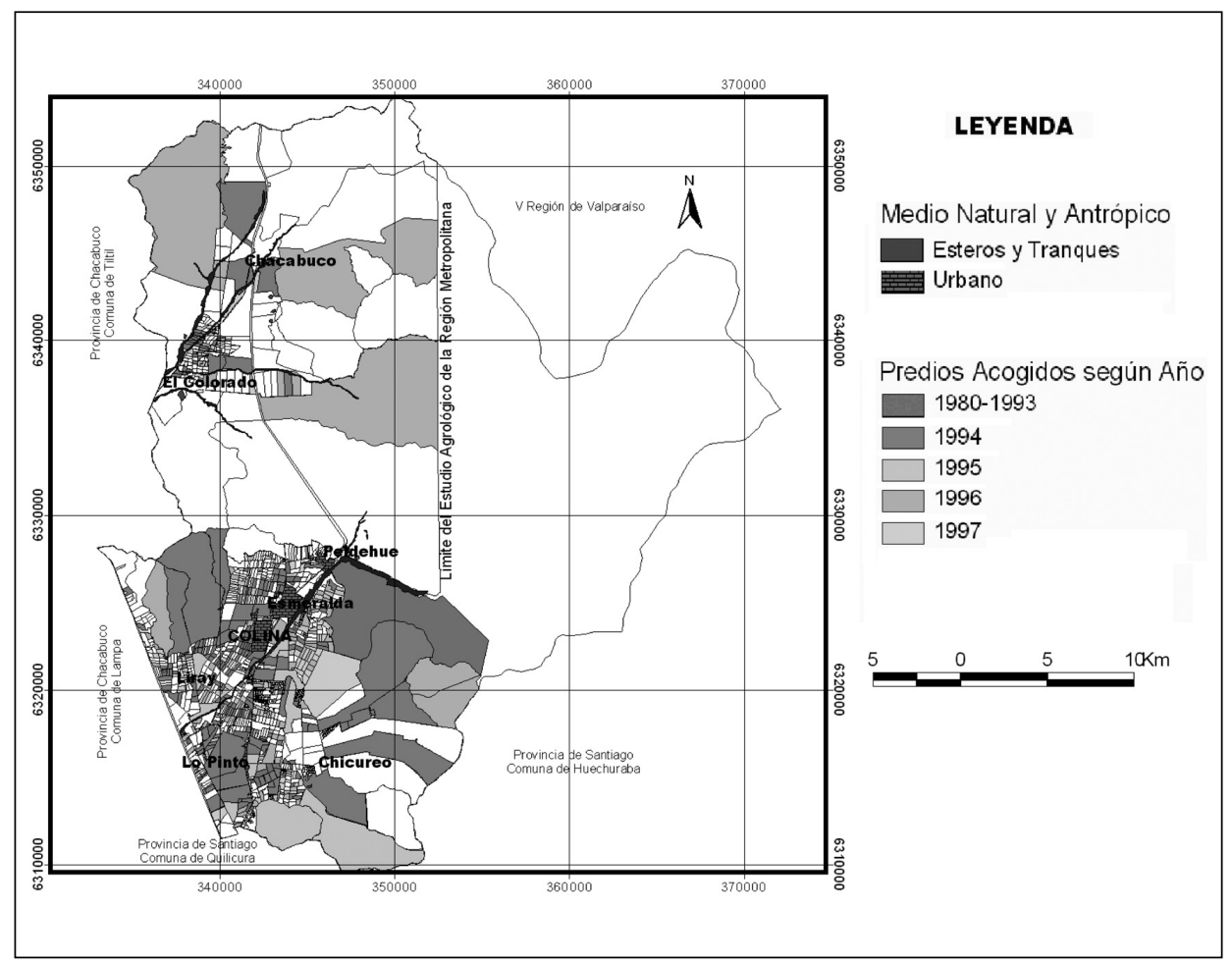

Fuente: datos elaborados por la autora sobre la base de CIREN 1997, archivos SAG y Conservador de Bienes Raíces de Santiago

MAPA 2. Predios acogidos al DL 3.516 en la comuna de Colina

\section{Estado de consolidación de los predios acogidos al DL 3.516}

El estado de consolidación alude a la presencia de obras de urbanización en los predios acogidos al DL 3.516/80, existiendo dos posibilidades: subdivisiones consolidadas y subdivisiones no consolidadas. Es una variable de gran relevancia en esta investigación ya que representa una certera aproximación a la comprobación de procesos de especulación sobre el mercado de la tierra. Los predios no consolidados constituyen la reserva del mercado de tierras con los que la especulación se manifiesta.

Actualmente se encuentra consolidada en la provincia de Chacabuco el 43 por ciento de la superficie total acogida. Si se analiza esta situación para cada 
una de las comunas que integran la provincia, se puede constatar que en la comuna de Colina, donde la consolidación es más evidente, se encuentra consolidada el 64,09 de la superficie total acogida en esa comuna. En tanto en la comuna de Lampa, se ha consolidado el 14,87 por ciento y en la comuna de Tiltil sólo el 0,79 por ciento de la superficie total acogida a esta ley. La tabla 2 da a conocer esta situación para las comunas de la provincia de Chacabuco y para otras comunas de la periferia del Gran Santiago.

Es posible identificar ciertas áreas de mayor desarrollo inmobiliario en la provincia de Chacabuco. Estas están localizadas preferentemente en la comuna de Colina, con el ejemplo emblemático de Chicureo. Luego, en Lampa con el desarrollo adquirido en las inmediaciones de Batuco, que está en pleno auge y que muestra un retardo respecto de lo ocurrido en Colina. Finalmente, y en menor medida, en Tiltil, por el desarrollo ocurrido en Caleu, ya que en el resto de la comuna prácticamente no hay ofertas por el momento.

Por otra parte, se puede vislumbrar ciertas tendencias a la localización de predios consolidados, cuya manifestación más evidente son las parcelas de agrado. Se identifican especialmente cercanas a las vías estructurantes de comunicación, así como también en ciertos núcleos de desarrollo inmobiliario marcados por la exclusividad (se manifiestan en rejas, presencia de vegetación desarrollada y sistemas de seguridad que impiden el paso a los no residentes).

Es claro que la consolidación se ha manifestado en mayor medida en la comuna de Colina y que aún quedan disponibles muchos predios con sus subdivisiones aprobadas que forman parte de la reserva para la especulación en el mercado de tierras. Muchos de los propietarios que se acogieron a la ley de subdivisión aún no han puesto en venta estas tierras, sino que lo harán en el momento más propicio desde el punto de vista económico, cuando el precio del suelo alcance un valor más conveniente, situación que aún no se ha verificado. Se comprueba así la especulación sobre el mercado de la tierra.

\section{COMENTARIOS}

El DL 3.516/80 se promulga en 1980 y ese mismo año comienza a aplicarse en las comunas de Colina y Lampa. En la comuna de Tiltil solo se aplica a partir del año 1994, año que coincide con la aparición del Plan Regulador Metropolitano de Santiago.

El DL 3.516/80 sobre subdivisión de predios rústicos produjo cambios en el paisaje de la provincia de Chacabuco que no estaban previstos. En primer lugar, produce una trama predial radicalmente distinta a la existente previo a 
la aplicación de esta ley, afectando a 1.592 predios que darían lugar a 73.526 lotes; esta cifra está indicando que cada predio se divide en promedio en 46 lotes, lo que conlleva a una atomización de la propiedad que viene a reafirmar su ineficacia para mantener en ellos un uso agrícola. Esto último, debido fundamentalmente a la pequeña magnitud de la superficie a utilizar a lo que se suma el hecho de que quienes compran estas parcelas son habitantes de la ciudad que buscan vivir gozando de las bondades del campo con las comodidades de la vida urbana. Cabe destacar que si los 73.526 lotes se consolidaran en su totalidad, se estaría involucrando a una población de alrededor de $309 \mathrm{mil}$ habitantes (cifra que resulta de la multiplicación del número de lotes por 4,2, que corresponde al número medio de personas por familia en Chile). Si ello se suma a la cifra de población de la provincia para el año 2002, de 132.798 habitantes, se llega a una población de 441.798 personas....

Cabe preguntarse acerca de la sustentabilidad del territorio para albergar a una población tal; si existen los recursos básicos necesarios; si se podrá dotar de los servicios e infraestructuras necesarias para que esa población tenga una adecuada calidad de vida; qué pasará con los habitantes tradicionales de ese territorio y sus identidades, con los agricultores y campesinos que están viendo sus modos de vida, costumbres y sus fuentes laborales afectadas por el cambio de su entorno; cómo se abordará la gestión de estos territorios y las externalidades que se generarán, especialmente aquellas relacionadas con la inequidad.

En cuanto a la distribución de los predios acogidos, hay una clara concentración en el sector sureste de la provincia de Chacabuco, siendo esta distribución para cada comuna la siguiente: en Colina se concentran en el sector sur de la comuna, sin discriminación de tamaño; en Lampa se concentran fundamentalmente en el sector oriente y afecta de preferencia a predios de tamaño mediano y pequeño, pudiéndose identificar una supeditación al relieve; en la comuna de Tiltil la distribución es aleatoria, sin discriminación de tamaño predial ni de relieve.

En lo referente a ciertos hitos respecto del proceso en las comunas de la provincia de Chacabuco, destaca en las comunas de Lampa y Tiltil el año 1996 como aquel en que un mayor número de dueños se acogen al DL 3.516/80; en cambio, en la comuna de Colina es el año 1994.

En cuanto a la consolidación de los predios acogidos al DL 3.516/80 en la provincia de Chacabuco, cabe destacar que el 43 por ciento de la superficie se encuentra consolidada. Para la comuna de Tiltil, la cifra de consolidación es marginal, de menos del 1 por ciento, en la comuna de Lampa, de alrededor del 15 por ciento y en la comuna de Colina, de casi el 65 por ciento de la superficie acogida al DL 3.516/80. Esto tiene una posible explicación en la distancia a 
la ciudad de Santiago y en el mayor desarrollo inmobiliario y vial que ha tenido Colina y que comienza a presentar Lampa.

Respecto a la calidad de los suelos afectados por el DL 3.516/80, el comportamiento en las tres comunas es diferenciado. En Tiltil la superficie de suelos arables y no arables bordea el 40 por ciento. En Lampa, la superficie arable también es de alrededor del 40 por ciento, sin embargo, la superficie no arable es de 17,07 por ciento. En Colina, en cambio, afecta al 62 por ciento de los suelos arables y al 90,07 por ciento de los suelos no arables. La importancia del efecto espacial del DL 3.516/80 radica en el cambio de uso del suelo que implica: si está consolidado, representa un cambio a uso urbano ya materializado. Si aún no se encuentra consolidado, puede hacerlo a futuro. De cualquier forma, el destino de estas tierras está ya trazado y no considera la vocación rural de estos espacios.

Al considerar la calidad de los suelos afectados por el DL 3.516/80 en la provincia de Chacabuco, que son las tierras efectivamente perdidas para las actividades silvoagropecuarias, se verifica que en la comuna de Tiltil la consolidación afecta principalmente a suelos no arables, en tanto en la comuna de Lampa las cifras de consolidación en suelos arables y no arables bordean el 5 por ciento. En la comuna de Colina, en cambio, están consolidados el 68,11 por ciento de los suelos arables acogidos.

Aún no se han perdido parte de los mejores suelos para la agricultura por la infiltración de la ciudad de Santiago al amparo del DL 3.516/80. Aunque, como ya se ha dicho, el destino de estas tierras ha sido trazado, cabe aún pensar en la protección que necesitan estos suelos y lo que es más importante, de los habitantes que moran en ellos y de sus modos de vida rurales tradicionales que están siendo afectados por el avance urbano. La comuna de Lampa, de acuerdo a estimaciones efectuadas en el PRMS, espera recibir en los próximos 30 años una población de 120.000 habitantes y para ello hay una serie de proyectos aprobados en la comuna. En tanto, en la comuna de Colina existen planes que estiman que Colina se convertirá en una ciudad de unos 200.000 habitantes hacia el año 2.015. Esto significa que en los próximos años, la población habrá crecido en más de 120.000 personas. Confirma esta afirmación la aprobación al proyecto de Modificación del Plan Regulador Comunal de Colina, tendiente a cambiar el uso del suelo en cerca de 750 hectáreas, actualmente consideradas como silvoagropecuarias, para proyectos de urbanización y la incorporación de las 12 comunas a partir de 2006 al PRMS.

Si desde el contexto regional la provincia de Chacabuco se vislumbra como el lugar de residencia de un alto monto de población, queda claro que no existe por parte de los organismos del Estado la preocupación necesaria por la 
conservación de los suelos con aptitud agrícola, que no cuentan con ninguna protección ni intención de conservación.

El DL 3.516/80 ha contribuido a la urbanización al margen de los instrumentos de ordenamiento territorial, cuyo objeto es precisamente "ordenar" el uso del suelo. Sin embargo, hay que precisar que ordenan los espacios urbanos y generan consecuencias poco amigables con "el orden" en los espacios rurales de su periferia, contribuyendo a la expansión de la ciudad en una forma urbana, cuando menos, diferente a lo que todos hasta aquí entendíamos como tal y que al parecer de la autora, corresponde a la ciudad infiltrada. Queda por delante la tarea de observar y estudiar las consecuencias de la última modificación al PRMS, que sin duda marcarán el destino de los espacios rurales periurbanos.

\section{CONCLUSIONES}

a) Se trata de un proceso inducido por el Estado a través de un organismo sectorial ajeno a propósitos relacionados con el desarrollo urbano.

b) El mercado inmobiliario encontró bajo este escenario un territorio propicio para maximizar la rentabilidad de sus proyectos, dado la incapacidad de los ministerios responsables del desarrollo urbano de regular estos procesos.

c) Asimismo, el desarrollo de las infraestructuras promovidas por el mismo Estado, permitieron rentabilizar los proyectos inmobiliarios, sin que las externalidades provocadas por sus promotores fueran debidamente compensadas, afectando seriamente la conectividad de algunas localidades rurales en el espacio local e intercomunal.

d) La inexistencia de políticas en materia de ordenamiento territorial, fundamentalmente debido a que las atribuciones sobre la materia se encuentran atomizadas en diferentes ministerios y organismos sectoriales que no terminan de coordinarse en forma adecuada, plantea un desafío no menor para el Estado en el propósito de promover un desarrollo urbano y territorial que no vaya en desmedro del desarrollo rural.

e) Las iniciativas en materia de proyectos de ley actualmente en curso, no permiten en forma satisfactoria visualizar un desarrollo urbano y territorial futuro donde se garantice a la población de los sectores rurales afectados tres objetivos básicos de la política pública: cohesión social, desarrollo económico y sustentabilidad ambiental.

Recibido: 20/01/2009

Aceptado: 03/03/2009 
BIBLIOGRAFÍA

Arenas, F., Hidalgo, R. y Coll, J. (2003): "Los nuevos modos de gestión de la metropolización". Santiago de Chile. Serie Geolibros 2. Instituto de Geografía, Facultad de Historia, Geografía y Ciencia Política, Pontificia Universidad Católica de ChileUniversidad de Toulouse le Mirail, Francia.

Arenas, F. e Hidalgo, R. (2002): "Asentamientos Humanos". En Universidad de Chile, Instituto de Asuntos Públicos, Informe país: Estado del medio ambiente en Chile.2002, pp. 353-386. Santiago, LOM Ediciones.

Ascher, F. (1995): "Dynamiques métropolitaines et enjeux socio-politiques". Futur Antérieur. París.

Brignardello, L. y Georgudis, B. (1997): "Geopodología de la cuenca de Santiago: Dimensión espacial de los problemas ambientales en los suelos". Santiago de Chile. En Norte Grande, 24:127-141.

Capel, H. e Hidalgo, R. (editores) (2006): Construyendo la ciudad del siglo xxi. Retos y perspectivas urbanas en España y Chile. Santiago de Chile. Serie Geolibros No 6. Instituto de Geografía Facultad de Historia, Geografía y Ciencia Política Pontificia Universidad Católica de Chile, Geocrítica Textos de Apoyo Departamento de Geografía Humana Universidad de Barcelona.

Centro de Información de Recursos Naturales (1997): Ortofotos escala 1:20.000 correspondientes a las comunas de Colina, Lampa y Tiltil. Santiago, 1997.

De Mattos, C. (1998): "Reestructuración, crecimiento y expansión metropolitana en las economías emergentes latinoamericanas". Santiago de Chile. En Economía, Sociedad y Territorio, vol. I, núm. 4, 1998, 723-754.

De Mattos, C. (1999): "Santiago de Chile, globalización y expansión metropolitana: lo que existía sigue existiendo". Santiago de Chile. En Revista EURE, vol. XXV, 76:29-56.

De Mattos, C. (2005): "Santiago de Chile: Metamorfosis bajo el nuevo impulso de modernización capitalista." Santiago de Chile. En De Mattos, C., Ducci, M., Rodríguez, A. y Yánez, G. editores: Santiago en la Globalización. Segunda edición. Coedición Ediciones Sur-EURE Libros. Pp 17-47.

Diario Oficial No 30.829 de 1/12/1980.

Dollfus, O. (1994): L'espace monde. Ed. Economica. París..

Ferrao, J. (1992): Servicos e inovacao. Novos caminhos para o desenvolvimento regional. Oeiras. Celta Editora.

Greene, M. y Soler, F. (2005): "Santiago: de un proceso acelerado de crecimiento a uno de transformaciones". Santiago de Chile. En De Mattos, C., Ducci, M., Rodríguez, A. y Yánez, G. editores: Santiago en la Globalización. Segunda edición. Coedición Ediciones Sur-EURE Libros.

Hidalgo, R. y Borsdorf, A. (2005): "Megaproyectos residenciales vallados en la periferia: ¿Barrios cerrados autosuficientes o nuevas ciudades?” Santiago de Chile. En Revista URBANO (5-12). 
Hidalgo, R. y Borsdorf, A. (2005): "La exclusión residencial y el desarrollo de la ciudad moderna en América Latina: de la polarización a la fragmentación. El caso de Santiago de Chile". En Geographicalia, 48 (5-29).

Hidalgo, R., Salazar, A., Lazcano, R., Álvarez, L. y Calderón, M. (2005): “Transformaciones socioterritoriales asociadas a proyectos residenciales de condominios en comunas de la periferia del Área Metropolitana de Santiago." Santiago de Chile. En Revista INVI No 54, Vol. 20 (104-133).

Instituto Nacional de Estadísticas (2002): Censo 2002. Santiago: Instituto Nacional de Estadísticas.

Ley General de Urbanismo y Construcciones, artículos 52 y 55.

Lincoln Institut of Land Policy (1996): Global City Regions: Searching for Commond Grund. Landlines, vol. 8, 1.

Miranda, C. (1997): "Expansión Urbana Intercensal del Gran Santiago 1875-1992". Santiago de Chile. En Estadística y Economía, (77-104).

Naranjo, G. (2002): Efectos de un instrumento de planificación en el periurbano de Santiago. Caso de estudio: comuna de Tiltil. Santiago de Chile. Tesis para optar al Grado de Magister en Asentamientos Humanos y Medio Ambiente. Instituto de Investigación y Postgrado. Facultad de Arquitectura, Diseño y Estudios Urbanos. Pontificia Universidad Católica de Chile.

Naranjo, G. (2004): Impacto territorial de las parcelas de agrado en el periurbano de Santiago. Caso de estudio: comuna de Lampa. Región Metropolitana de Santiago. Santiago de Chile. Informe Final del Proyecto DIPUC No 2003/11E2.

Naranjo, G. (2006): Impacto territorial del DL 3.516/80 en el periurbano de Santiago. Caso de estudio: Comuna de Colina. Santiago de Chile. Informe Final del Proyecto DIPUC No 2005/09PI.

Naranjo, G. (2006): "Efectos del Plan Regulador Metropolitano de Santiago en el ordenamiento de los espacios periurbanos". En: Capel, H. e Hidalgo, R. (editores): Construyendo la ciudad del siglo xxi. Retos y perspectivas urbanas en España y Chile. Santiago de Chile. Serie Geolibros N 6 Instituto de Geografía. Facultad de Historia, Geografía y Ciencia Política. Pontificia Universidad Católica de Chile-Geocrítica. Textos de Apoyo Departamento de Geografía Humana Universidad de Barcelona. Pp 293-306.

Salazar, A. (2007): "La periurbanización en la recomposición de los espacios rurales metropolitanos: Santiago de Chile". En: De Mattos, C. e Hidalgo, R. (editores): Santiago de Chile: Movilidad espacial y Reconfiguración Metropolitana. Colección EURE-LIBROS Serie GEOlibros $\mathrm{N}^{a}$ 8. Instituto de Estudios Urbanos y Territoriales-Instituto de Geografía, Pontificia Universidad Católica de Chile. 


\section{RESUMEN}

El DL 3.516/80 permite la división de predios rústicos hasta 0,5 hectárea. Su aplicación en la periferia de Santiago ha generado la urbanización del campo al margen de los instrumentos de ordenamiento territorial, que regulan solo los espacios urbanos.

Santiago sufrió una fuerte expansión hasta que en 1994, el Plan Regulador Metropolitano de Santiago (PRMS), fija el límite urbano. Su aparición genera procesos de subdivisión predial y de especulación sobre la tierra, al amparo del DL 3.516/80.

Esta investigación estudia el Impacto territorial del DL 3.516/80 y su relación con el PRMS en la periferia de Santiago de Chile. Mediante un análisis bibliográfico y cartográfico, entrevistas a agentes locales claves y el uso del SIG, se determina la superficie afectada por el DL 3.516, la calidad de sus suelos y la superficie perdida para la agricultura por la urbanización del campo, con lo que se constituye la "ciudad infiltrada".

PAlabras ClaVE: periurbano; ciudad infiltrada; expansión metropolitana.

\section{Abstract}

The DL 3.516/80 allows the division of rustic properties up to 0,5 hectares. Its application in the outskirts of Santiago has generated the urbanization of the countryside not considering the existing territorial planning instruments, which only regulate urban spaces.

Santiago suffered a strong expansion until 1994 when the Metropolitan Regulation Plan of Santiago (PRMS in Spanish), fixed the urban boundary. Its appearance generated the subdivision of fields and the speculation of land, under the DL 3.516/80.

This investigation studies the territorial impact of the DL 3.516/80 and its relation with the PRMS on the outskirts of Santiago, Chile. Through a bibliographic and cartographic analysis, interviews to key local agents and using GIS, the area affected by the DL 3.516/80 is determined, the quality of its soils and the area lost by agriculture due to the urbanization of the countryside, which generates the "infiltrated city".

KEY WORDS: periurban; infiltrated city; metropolitan expansion.

\section{RÉSUMÉ}

Le DL 3516/80 permet la division des terres rustiques jusqu'à 0,5 ha. Son application dans la périphérie de Santiago a généré l'urbanisation de la campagne indifféremment des instruments d'ordre territorial, qui régulent seulement les espaces urbains.

Santiago a souffert une forte expansion jusqu'en 1994 quand le Plan Régulateur Métropolitain de Santiago (PRMS en espagnol), fixe la limite urbaine. Son apparition en- 
gendre des procès de subdivision des propriétés et de la spéculation sur la terre, sous le DL 3.516/80.

Cette investigation étudie l'impact territorial du DL 3.516/80 et sa relation avec le PRMS dans la périphérie de Santiago du Chili. À travers d'une analyse bibliographique et cartographique, interview avec des agents locaux clés et l'utilisation des SIG, la superficie affectée par le DL 3.516/80 est déterminée, la qualité de ses sols et la superficie perdue par l'agriculture à cause de l'urbanisation de la campagne, ce qui constitue "la ville infiltrée".

MoTS CLÉS: periurbain; ville infiltrée; expansion métropolitaine. 